\title{
Can a single low-intensity premature stimulus induce ventricular arrhythmias in the normal heart?
}

\author{
Stefano Rossi, ${ }^{1}$ Silvana Baruffi, ${ }^{1}$ Sergio Callegari, ${ }^{2}$ Luca Carnevali, ${ }^{3}$ Domenico Corradi, ${ }^{4,5}$ \\ Michele Miragoli, ${ }^{5}$ Ezio Musso, ${ }^{1}$ Andrea Sgoifo, ${ }^{3}$ Mimosa Trombini, ${ }^{1}$ Emilio Macchi, \\ ${ }^{1}$ Department of Biosciences, University of Parma; ${ }^{2}$ Department of Cardiology, Vaio Hospital, \\ Fidenza; ${ }^{3}$ Department of Neurosciences, University of Parma; ${ }^{4}$ Department of Biomedical, \\ Biotechnological and Translational Sciences, University of Parma; ${ }^{5}$ Centre of excellence for \\ Toxicological Research, University of Parma, Italy
}

\begin{abstract}
Previously, we observed that a single low-intensity premature ventricular stimulation could occasionally induce spontaneous ectopic beats in normal rat hearts. Possible hypothesis for the arrhythmia is that a premature beat can encounter a zone of conduction block to initiate reentry. However, enhanced dispersion of repolarization, a necessary condition for initiation of reentry, is unlikely to be present in normal myocardium. Thus, the main objective of the present study was to perform detailed pace mapping measurements in normal ventricular myocardium with a view to identify pacing sites and critical coupling intervals which could induce spontaneous ectopic beats and to characterize the reentrant circuits.
\end{abstract}

\section{Introduction}

In previous experiments, we observed that single low-intensity premature stimuli could occasionally induce ectopic beats in normal

Correspondence: Emilio Macchi, Department of Biosciences, University of Parma, Parco Area delle Scienze 11A, 43124 Parma, Italy.

Tel. +39.0521 .902111 - Fax: +39.0521 .347038 .

E-mail: emilio.macchi@unipr.it

Key words: premature stimulation, conduction block, reentry circuit, arrhythmia.

Acknowledgements: we acknowledge Riccardo Labadini (University of Parma, Italy) for his technical assistance.

Funding: authors received financial support from Fondazione CARIPARMA, Bilancio 2010.

(C) Copyright S. Rossi et al., 2014

Licensee PAGEPress, Italy

Journal of Biological Research 2014; 87:2128

doi:10.4081/jbr.2014.2128

This article is distributed under the terms of the Creative Commons Attribution Noncommercial License (by-nc 3.0) which permits any noncommercial use, distribution, and reproduction in any medium, provided the original author(s) and source are credited. rat hearts. This event occurred while assessing local effective refractory period (ERP) by pacing the ventricles with a train of eigth basic stimuli (S1) followed, at the same location, by a premature stimulus (S2). Surprisingly, by repeating the premature stimulation at the same site and with the same coupling interval, the ectopic beats were consistently induced. Based on these observations, a possible hypothesis for the arrhythmia is that the premature beat acts as an initiating trigger which, in the presence of a suitable substrate, leads to reentrant excitation. ${ }^{1}$

It is commonly known that single, strong electrical stimuli delivered through a relatively small electrode can, when properly timed, induce ventricular arrhythmias in the normal heart, whereas stimuli of low intensity usually do not. ${ }^{2}$ Even when two to five successive low-intensity premature stimuli, i.e. stimuli with shorter coupling intervals than that of the basic rhythm, are applied to the normal ventricle, ventricular arrhythmias are rarely induced. ${ }^{2}$ It is likely that a key element, deciding whether or not electrical stimuli applied to the normal heart result in sustained repetitive activity, is a certain degree of inhomogeneity in the recovery of excitability of individual cardiac cells, meaning that at a particular instant some fibers are totally inexcitable, i.e. absolutely refractory, others have partially recovered their excitability, i.e. relatively refractory, and still others have completely recovered their excitability. Possible conditions for the arrhythmia is that the premature beat can encounter a zone of unidirectional conduction block to initiate reentry. ${ }^{1} \mathrm{~A}$ key requirement for the induction of reentry is the presence of unidirectional block. Usually, reentry occurs following a premature impulse, either occurring spontaneously or induced by a low-intensity stimulus. When properly timed, this impulse will be blocked in regions with a long refractory period, and slowly conducted around the zone of block in areas with shorter refractory periods to return to the point of origin resulting in reentrant excitation (Figure 1). The normal ventricular myocardium shows some inhomogeneity in the recovery of excitability, the difference between the shortest and longest refractory period, also called dispersion in refractoriness, being in the order of $40 \mathrm{~ms}^{2}{ }^{2}$ Furthermore, differences between the refractory periods of ventricular myocardium and the specialized conducting system, or Purkinje system, are even larger. ${ }^{3-5}$ Still, it is very unusual for single low-intensity premature stimuli to induce ventricular arrhythmias in normal myocardium. Consequently, conditions in which the dispersion in refractoriness is increased are associated with a greater likelihood for reentrant excitation to occur. Two of these conditions are enhanced activity of the sympathetic nervous system that results in, among others, an increase in the heart rate and acute regional ischemia, when sudden occlusion of a branch of a coronary artery results in an arrest of blood flow to part of the ventricles. 
Alternatively, a mechanism for the initiation of reentry by strong electrical stimulation from a point electrode was first proposed by Winfree, ${ }^{6}$ termed as the pinwheel experiment or singular point hypothesis, and later as the critical point hypothesis by Frazier et al. ${ }^{7}$ The mechanism, illustrated in Figure 2A, proposes that a premature electrical stimulus (S2) applied from a point electrode at a critical time $\mathrm{T}_{\mathrm{c}}$ during the repolarization phase of a rectilinear (S1) wave, such as a sinus beat, depolarizes a spatial domain of critical size $\mathrm{A}_{\mathrm{c}}$.

The S2 excitation cannot propagate upwardly in the same direction of the $\mathrm{S} 1$ beat, since the tissue in this region has not yet recovered excitability, but can propagate downward where the tissue has already recovered. This unidirectional conduction block of the $\mathrm{S} 2$ beat produces two singularities (wave breaks), which subsequently turn inward and attempt to form counterrotating spiral waves. Figure-of-eight reentry results if the two spiral wave tips have enough room to form a central common conduction pathway. However, if the S2 depolarizes a spatial domain smaller than the critical area, or if the S2 is given too early or too late, the two spiral wave tips will collide and annihilate (annihilation occurs if the 2 dashed circles in Figure 2A intersect). For a given S2 stimulation strength, there is a range of coupling intervals for which reentry can be induced, which is called the vulnerable period; the range of both coupling intervals and stimulus intensities inducing reentry is called the vulnerable domain. Subsequently, Roth ${ }^{8}$ extended the pinwheel experiment to the more realistic case of bidomain tissue (in
A

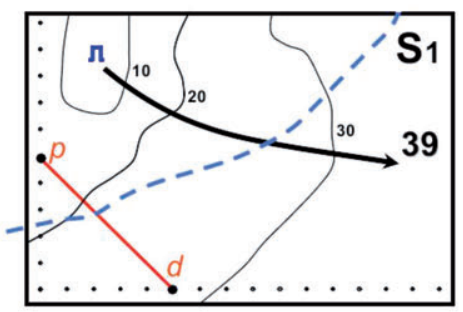

B

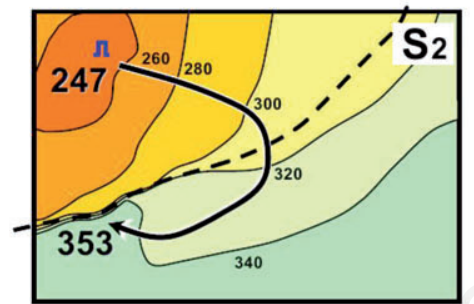

C

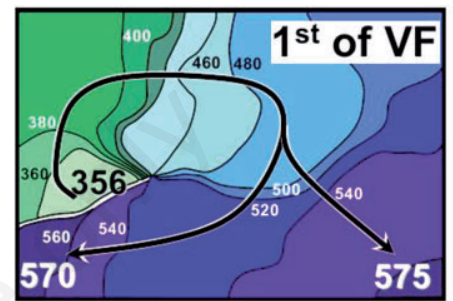

D

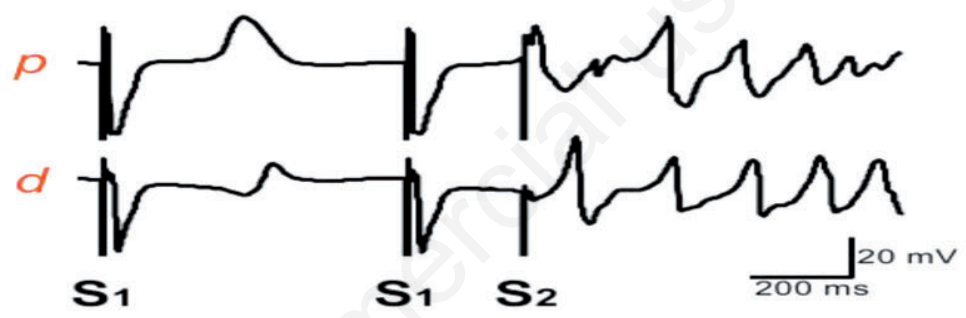

Figure 1. Differentially perfused myocardial regions in a Langendorff perfused pig heart. A) Left of the dashed line is the proximal tissue, perfused with pinacidil (shorter repolarization); right is the distal tissue perfused with sotalol (longer repolarization). Maximum gradient of repolarization is $120 \mathrm{~ms}$ across the border of the dashed line. Basic stimulation S1 (cycle length, $450 \mathrm{~ms}$ ) leads to smooth activation of the myocardium. B) Premature activation S2 demonstrates an area of block forcing the activation front to curve. The activation wave arrives at the distal side of the line of block at $353 \mathrm{~ms}$. C) At that time, the local shorter repolarization has ended and the activation wave fully reenters the proximal area, causing the first cycle of ventricular fibrillation. D) Proximal and distal tissue waves as for S1 and

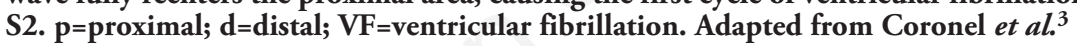
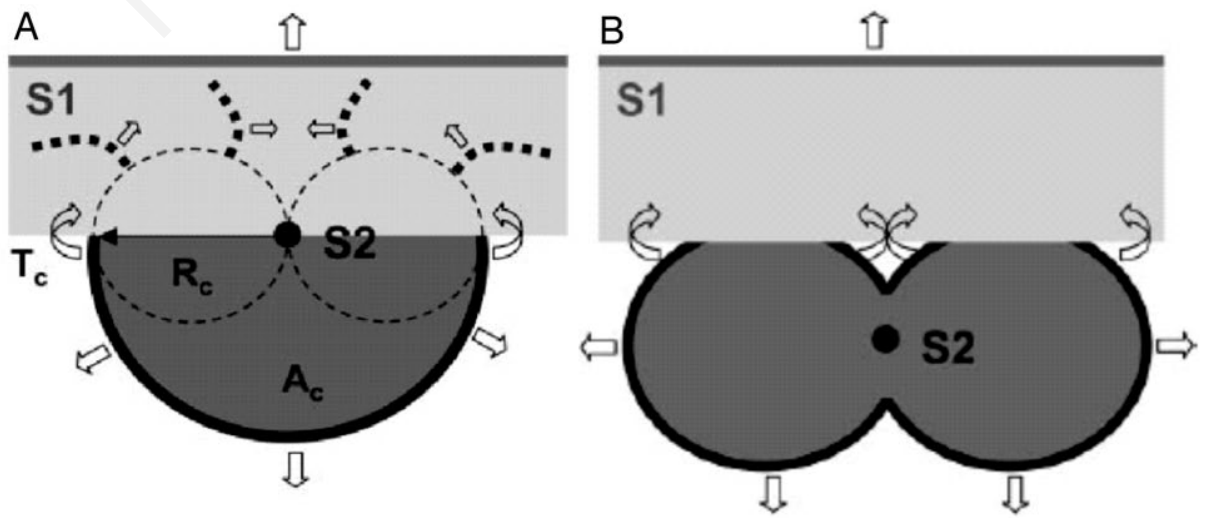

Figure 2. Schematic illustration of the pinwheel experiment. A) Winfree's pinwheel experiment ${ }^{6}$ where $S 1$ is a planar wave propagating upward and $S 2$ is delivered at a critical time from a point electrode that depolarizes a critical circular domain with a critical radius. Two singularities are generated at the intersection of the circle's edge with the wave back of S1, which turn inward to form 2 spiral waves and figure-of-eight reentry. B) Example of Roth's extension ${ }^{8}$ of Winfree's pinwheel experiment to bidomain tissue, in which virtual electrode formation creates 4 singularities that can induce quatrefoil reentry. $T_{c}=c$ critical time; $A_{c}=c r i t i c a l$ circular domain; $R_{c}=c r i t i c a l$ radius. 
which both intracellular and extracellular resistivities are represented). He proposed that the shock creates virtual electrodes, consisting of cathodal and anodal regions in a dog bone pattern (Figure 2B), which would affect the size of the vulnerable period and the number of rotors created (quatrefoil reentry).

Ultimately, most of the conditions which are known to favor reentry mechanisms were not present (strong electrical stimuli) or unlikely to be present (enhanced dispersion of refractoriness) in our experimental animals. Accordingly, the mechanisms underlying the origin of the ectopic beats induced by single low-intensity premature stimuli remain still elusive. Therefore, the main objective of the present study was to characterize pacing sites and critical premature intervals which can induce ectopic beats in the normal heart and to identify their arrhytmogenic substrate. To specify temporal and spatial properties of a possible reentrant excitation, we performed high resolution potential mapping during sequential premature pacing of multiple epicardial sites in normal rats. In order to evaluate whether spatial dispersion in recovery of excitability might be important for the occurrence of unidirectional block, we also measured the ERP at each pacing site. These measurements were then related with the spread of excitation during the onset of the arrhythmia.

\section{Materials and Methods}

Unipolar electrograms (EGs) were recorded from the anterior ventricular surface of 20 in vivo adult rats using a $8 \times 8,1 \mathrm{~mm}$ resolution, epicardial electrode array during sinus rhythm (SR) and premature stimulation. At 18 evenly distributed array electrodes, regular trains of low-intensity stimuli (two times diastolic threshold) were followed by a premature stimulus (eight times diastolic threshold) at progressively shorter coupling intervals, until ERP was reached.

\section{Results and Discussion}

Gradual increase in stimulus prematurity determined a corresponding increase in latency in impulse initiation as well as decrease in anisotropic conduction velocity. Ectopic beats were induced when pacing was performed at fixed sites of right (RV) and left ventricle (LV) and at well-defined coupling intervals proximal to ERP. Ectopic activation patterns were characterized by the appearance, on the paced ventricle, of epicardial breakthrough points (BTPs) which gave rise to wavefronts rapidly propagating in all directions. The location of BTPs was either coincident with SR BTPs or new BTPs could appear. For pacing sites located on the epicardial projection of the septum, BTPs could appear either on the RV or LV. Regions of critical degree of conduction and reentry were not observed on the epicardial surface, consistent with the measured uniform ERP spatial distribution.

\section{Conclusions}

The appearance of BTPs during the ectopic activation patterns is the epicardial manifestation of wavefronts originating from underlying Purkinje fiber-ventricular muscle junctions (PVJs). Thus, our findings suggest that a critically premature beat can encounter an appropriate substrate for unidirectional block and reentrant excitation at underlying PVJs where intrinsically different action potential durations are normally present at the two sides of the PVJ due to appreciably longer repolarization time of Purkinje fibers. ${ }^{4,5}$ Exactly the same issue would face a local spontaneous excitation in normal myocardium, such as a premature ventricular complex originating at a critical time during the repolarization phase of a sinus beat.

\section{References}

1. Kléber AG, Rudy Y. Basic mechanisms of cardiac impulse propagation and associated arrhythmias. Physiol Rev 2004,84:431-88.

2. Janse MJ. Vulnerability to ventricular fibrillation. Chaos 1998;8:149-56.

3. Coronel R, Wilms-Schopman FJ, Opthof T, Janse MJ. Dispersion of repolarization and arrhythmogenesis. Heart Rhythm 2009;6:53743.

4. Myerburg RJ, Stewart JW, Hoffman BF. Electrophysiological properties of the canine peripheral A-V conducting system. Circ Res 1970;26:361-78.

5. Mendez C, Mueller WJ, Merideth J, Moe GK. Interaction of transmembrane potentials in canine Purkinje fibers and at Purkinje fiber-muscle functions. Circ Res 1969;24:361-72.

6. Winfree AT. Electrical instability in cardiac muscle: phase singularities and rotors. J Theor Biol 1989;138:353-405.

7. Frazier DW, Wolf PD, Wharton JM, et al. Stimulus-induced critical point. Mechanism for electrical initiation of reentry in normal canine myocardium. J Clin Invest 1989;83:1039-52.

8. Roth BJ. The pinwheel experiment revisited. J Theor Biol 1998;190:389-93. 